\title{
Design and Synthesis of an Ultra Wide Band FSS for mm-Wave Application via General Regression Neural Network and Multiobjective Bat Algorithm
}

\author{
Miércio C. A. Neto ${ }^{1}$ (D) Jasmine P. L. Araújo ${ }^{1}$ (D) Raimundo J. S. Mota ${ }^{1}$ (D) Fabrício J. B. Barros ${ }^{1}$ (D), \\ Flávio H. C. S. Ferreira ${ }^{1}{ }^{\mathbb{D}}$, Gervásio P. S. Cavalcante ${ }^{1} \mathbb{D}$, Bruno S. L. Castro ${ }^{2} \mathbb{B}$ \\ ${ }^{1}$ Institute of Technology, Faculty of Electrical and Biomedical Engineering, Federal University of Pará, Brazil \\ miercio@ufpa.br,jasmine.araujo@gmail.com,raimota@ufpa.br,fbarros@ufpa.br, \\ henryferreira014@gmail.com,gervasio@ufpa.br \\ ${ }^{2}$ Faculty of Computer Science, Castanhal University Campus, Federal University of Pará, Brazil \\ brunoslc21@gmail.com
}

\begin{abstract}
In this work is presented a hybrid bioinspired optimization technique that associates a General Regression Neural Network (GRNN) with the Multiobjective Bat Algorithm (MOBA), for the design and synthesis of the Frequency Selective Surfaces (FSS), aiming its application in data communication systems by diffusion of millimeter waves, specifically, in the IEEE 802.15.3c standard. The designed device consists of planar arrangements of metallizations (patches), diamond-shaped, arranged over a RO4003 substrate. The FSS proposed in this study presents an operation with ultra-wide band characteristics, its patch designed to cover the range of $40.0 \mathrm{GHz}$ at $70.0 \mathrm{GHz}$, i.e., $30.0 \mathrm{GHz}$ bandwidth and 60.0 GHz resonance. The upper and lower cutoff frequencies, referring to the transmission coefficient's scattering matrix (dB), were obtained at the cutoff threshold at $-10 \mathrm{~dB}$, to control the bandwidth of the device.
\end{abstract}

Index Terms - hybrid technique, general regression neural network (GRNN), multiobjective bat algorithm (MOBA), Frequency Selective Surfaces (FSS).

\section{INTRODUCTION}

Evolutions in computational methods have made possible substantial advancements in engineering and industrial researches [1]. In these areas, the employment of computational techniques is intensifying for simulation purposes, and to obtain certain system parameters for investigated devices. However, the ever-growing demand for precision and the rise of complexity of devices result in a simulation process that takes longer, for the evaluation of a single criterion can consume several hours, or even days or weeks [2] - [3]. Therefore, a method that can minimize simulation time and increase optimization is desired, saving not only time but money.

In this context, Bioinspired Computing (BIC) presents itself as precise and efficient where often traditional computational methods fail, and consists of a new mechanism to make up for the difficulties imposed on the development of projects [2] - [3]. Thus, this work presents studies about one of the most utilized BIC algorithms, the Multiobjective Bat Algorithm (MOBA), proposed in 
Journal of Microwaves, Optoelectronics and Electromagnetic Applications, Vol. 18, No. 4, December 2019531 DOI: http://dx.doi.org/10.1590/2179-10742019v18i41729

2011 by Xin-She Yang [4].

The electromagnetic (EM) wave control through surfaces or border layers is a subject of great interest for researches in the field of applied electromagnetism. At this scenario, the study of Frequency Selective Surfaces (FSS) is highlighted [2], [3], [5] - [9], due to their capacity to effectively control characteristics such as frequency response magnitude, polarization and wave propagation phase in specific frequency bands [10]. This justifies the development of new techniques for the analysis, project and modeling of novel FSS geometrical shapes for applications in different patterns of frequency spectrums and systems [11].

In this way, this paper presents a Multiobjective Evolutionary Algorithm (MOEA), based on MOBA [4]. Initially, an EM investigation has been conducted on a new geometry for the FSS filter, named the Diamond-shaped Patch FSS, in which computational simulations utilizing the software HFSS were made, applying the Finite Element Method (FEM) for complete-wave analysis of EM properties for resonant structures. The following step in the process of planning and synthesis of the structure is the optimization of the unit-cell's geometric dimensions, with the goal of tuning its resonant frequency at $f_{r}=60.0 \mathrm{GHz}$ and a bandwidth of $\mathrm{BW}=30.0 \mathrm{GHz}$, for applications in frequency spectrums specified at the IEEE 802.15.3c standard [12].

The optimization process basically aims at a minimal computational effort, as well as maximizing advantages of the project [13]. That is, it searches for solutions that result in the minimal and maximal values of the cost function (or loss function). The methodology applied to the optimization process shown in this paper includes a General Regression Neural Network (GRNN) [14], that is trained by EM data calculated through the chosen numerical method - in this case, FEM. The GRNN becomes responsible for the analysis of the Diamond-shaped FSS and its EM properties, and following this, it creates a search space denominated Region of Interest (RoI). Within this region, the MOBA algorithm conducts a search for the best solutions, that is, the ones who attend the requisites of the cost function - thus characterizing this technique as a hybrid.

In the state-of-the-art, there is a vast literature in which is possible to verify that multiobjective, bioinspired hybrid optimizations are capable to provide faster convergence for solving the cost function. They also make a substantial reduction to demanded time for computational processing possible, as well as providing greater flexibility and accuracy of obtained results [2], [3], [15] - [18].

Such optimization techniques are greatly explored in projects involving microwave propagation, but less used in systems operating at higher frequencies. So, the objectives of the synthesis process present in this research are bandwidth and resonance tuning for the proposed FSS, which is to be utilized for applications in mm-Wave broadcast systems, following the IEEE 802.15.3c standard. As said previously, the objective is to make this device capable of resonating at $f_{r}=60 \mathrm{GHz}$ with a BW $=$ $30 \mathrm{GHz}$ bandwidth. This spectrum is being extensively explored for possible applications in the novel fifth generation of wireless communication, the 5G system [19] - [24].

The contribution of this research is the development of a novel hybrid technique, that associates Brazilian Microwave and Optoelectronics Society-SBMO received 14 Apr 2019; for review 25 Apr 2019; accepted 30 Aug 2019 Brazilian Society of Electromagnetism-SBMag 
GRNN to MOBA, for applications in the mm-Wave band, that possibly can be utilized to the new 5G standards. As verification for the hybrid technique's calculated results, other simulations involving FEM and the Finite Integration Technique (FIT) were conducted by the software CST®. The FSS optimized parameters using MOBA, are: $\mathrm{h}=0.5 \mathrm{~mm}$; $\mathrm{Tx}=1.85 \mathrm{~mm}$ and $\mathrm{Ty}=3.7 \mathrm{~mm} ; \mathrm{Wx}=1.75$ $\mathrm{mm}$ and $\mathrm{Wy}=3.5 \mathrm{~mm}$. Satisfactory, concordant results were observed at all calculations, assuring the developed code's stability and precision and the results displayed throughout this paper.

\section{THE BAT'S ECHOLOCATION ALGORITHM}

The bat algorithm was first introduced by Yang [25] in 2010, and it is based on echolocation, or the location by echo during flight that is executed by many species of bats. On flight, bats emit ultrasonic waves, generally in the $25 \mathrm{kHz}$ to $150 \mathrm{kHz}$ spectrum, through the nostrils or the mouth (this varies from species to species). These waves hit obstacles in the environment and return in the form of an echo with a frequency higher than the emitted one, given as the velocities of the bat and the echo sum up.

Based on the delay time and relative frequency of the echoes, bats can tell if there are obstacles in the way, just as their distances, shapes and relative velocities. It is particularly useful for hunting flying insects - however, other bat species with different eating habits also utilize this feature greatly.

For the sake of simplicity, the following rules were idealized for the development of the bat algorithm [25]:

i. All bats make use of echolocation to perceive and calculate distance, as well as recognizing the difference between your food/prey and spatial conditions of the environment;

ii. Bats run through the search space with a velocity, $v_{i}$, at a certain position $x_{i}$ (where $x_{i}$ is the solution for the problem), with a fixed frequency. $f_{\text {min }}$, and a varying wavelength $\lambda$ (or frequency $f$ ), and with an amplitude for the emitted sound $A_{\min }$ when hunting for prey. They can be automatically adjust the wavelength (or frequency) of their emitted pulses and adjust the pulse emission rate, $r \in[0,1]$, depending on the proximity of the target.

Even though the amplitude may vary in many ways, it is assumed that the variation is within the range of $\left[A_{\min }, A_{\max }\right]$.

\section{A. Computational movement of bats}

At the start of the code, a population of bats is randomly generated respecting the positioning $x_{i}$ and velocity $v_{i}$ in an n-dimensional search area.

Just as it occurs in the Genetic Algorithm (GA), the population is then evaluated and classified according to its aptitude to solve the specified cost function.

The new solutions, $x_{i}^{t}$, and velocities, $v_{i}^{t}$, for each iteration, $t$, are given by [4]:

Brazilian Microwave and Optoelectronics Society-SBMO received 14 Apr 2019; for review 25 Apr 2019; accepted 30 Aug 2019 $\begin{array}{lllll}\text { Brazilian Society of Electromagnetism-SBMag } & \text { ( ) } 2019 \text { SBMO/SBMag } & \text { (cc) BY } & \text { ISSN 2179-1074 }\end{array}$ 


$$
\begin{gathered}
f_{i}=f_{\text {min }}+\left(f_{\text {max }}-f_{\text {mín }}\right) \beta \\
v_{i}^{t}=v_{i}^{t-1}+\left(x_{i}^{t}-x_{*}\right) f_{i} \\
x_{i}^{t-1}=x_{i}^{t-1}+v_{i}^{t}
\end{gathered}
$$

in which $\beta$ is a random scalar value with uniform distribution, $\beta \in[0,1]$, and $x_{*}$ is the best localization (solution) found after comparing between all other solutions from other bats in the current iteration. Initially, the frequency of each bat is randomly distributed between $\left[f_{\min }, f_{\max }\right]$.

With up-to-date frequency parameters, velocity and positioning of bats, the next step is to evaluate the pulse emission rate for each bat. Following this, a comparison is drawn between all pulse emission rates and a random noise (generated by a rand function). If the rate of a bat possesses an inferior value than the noise's magnitude, it is because said bat is distant from the desired solution. Thusly, a local search is to be conducted, but the implementation for this strategy can be done in various ways, according to its adequacy to the project.

The local search, for this case, picks one solution amongst the best ones, and a new solution for every bat is generated locally through a process called "random walk":

$$
x_{\text {new }}=x_{\text {previous }}+\xi A^{t}
$$

in which $\xi \in[-1,1]$ is a random number, evenly distributed, and $A^{t}$ is the average value of the magnitude for all bats within an iteration $\boldsymbol{t}$.

Similar to Particle Swarm Optimization (PSO), the procedure of updating the velocities and positions of bats is similar to the rhythm and amplitude control pattern for particle movements. However, the bat algorithm considers a balanced combination of the PSO and the sound intensity search, controlled by volume and pulse rate [25]. It compares the previous solution to the current one, to select the best solution (with a greater aptitude value). Beyond this, it also compares the magnitude of the pulse (volume) with a random volume value (rand). If the random volume is weaker than the actual volume value for a bat, $A_{i}$, this means that this bat (solution) is drawing closer to the prey/target (best solution).

With that in consideration, solutions are accepted and the emission rates $r_{i}$ and magnitudes $A_{i}$ are updated for each iteration $\boldsymbol{t}$, according to these expressions:

$$
\begin{gathered}
r_{i}^{t+1}=r_{1}^{0}[1-\exp (-\gamma t)] \\
A_{i}^{t+1}=\alpha A_{i}^{t}
\end{gathered}
$$

in which $\alpha$ and $\gamma$ are constant at interval $0<\alpha<1$ e $\gamma>1$. That is:

$$
A_{i}^{t} \rightarrow 0 \text {, e } r_{i}^{t} \rightarrow r_{i}^{0} \text {, with } t \rightarrow \infty
$$


As the bat is within proximity of its target, the magnitude for the emitted pulse (A) diminishes, while its emission rate $(r)$ rises. Therefore, $A_{\min }=0$ is the instance when the bat has arrived at its target and temporarily stopped emitting any sound. So, the parameters $A$ and $r$ are updated solely if any new solutions are better than previous ones - which means the bats are moving within the search area along the optimal solution.

For practical, implementational purposes, $\left[0, f_{\max }\right]$ is utilized, and the emission rate is within the range $r \in[0,1]$, where " 0 " means there is no emission and " 1 " characterizes a maximum value of emission.

\section{B. Multiobjective bat algorithm}

The Multiobjective Bat Algorithm (MOBA) has been reformulated by Xin-She Yang and presented in 2010 [4]. Given that it is an algorithm that deals with multiobjective problems, two or more solutions are considered, and some of said solutions can be better than others in relation to all considered objectives - these are called non-dominated solutions. Generally, multiobjective optimization problems require an alternative definition of the "optimal values", or reference values, that can be approximated by "optimality" fronts. The Pareto Front [26] is the most applied parameter to this sort of problem-solving.

As the optimization problem exposed in this work refers to the minimization of the difference between the project's objectives and the constant optimal solutions at the Pareto Front, the following restrictions are considered [2] - [3]:

$$
\operatorname{Max}\left\{f_{1}(x), f_{2}(x), \ldots, f_{n}(x)\right\} \quad \therefore \quad \mathrm{X} \in \Omega \subset \mathbb{R}^{m}
$$

If $\mathrm{X}_{0} \in \Omega$, such as $f_{i}\left(x_{0}\right) \geq f(x) \forall \mathrm{X} \neq \mathrm{X}_{0} \in \Omega$, for some value of $\boldsymbol{i}$, therefore $\mathbf{X}_{\mathbf{0}}$ is said to be non-dominated in $\Omega$. All the $\mathbf{X}_{\mathbf{0}}$ points that satisfy the restriction above are part of, and denominate, the Pareto Front.

On the relationship of domination of results, if $x_{1}$ and $x_{2} \in \mathbb{R}$, where $\mathbb{R}$ is a region of achievable solutions, $x_{2}$ dominates $x_{1}$ if $f\left(x_{2}\right)$ is taken as being partially bigger, or bigger, than $f\left(x_{1}\right)$, that is,

$$
f_{1}\left(x_{1}\right) \leq f_{i}\left(x_{2}\right), \forall i=1,2, \ldots, n
$$

and,

$$
f_{1}\left(x_{1}\right)<f_{i}\left(x_{2}\right), \exists i=1,2, \ldots, n
$$

in case there is no $x_{i} \in \mathbb{R}$ that can dominate $x_{2}$, therefore $x_{2}$ is assumed to be a Pareto optimal solution.

Figure 1 shows an example of this relationship of domination, for the optimization problem investigated in this paper. It is possible to verify cross-shaped markers that have the purpose of identifying the bandwidth and resonant frequency for distinct iterations executed by the algorithm. Also, two of these markers are highlighted, one surrounded by a square and the other by a circle, 
representing the dominated solution (worst case) and the non-dominated solution (best case) respectively. As it exerts domination over all others, The Pareto Front's optimal solution is always the non-dominated one.

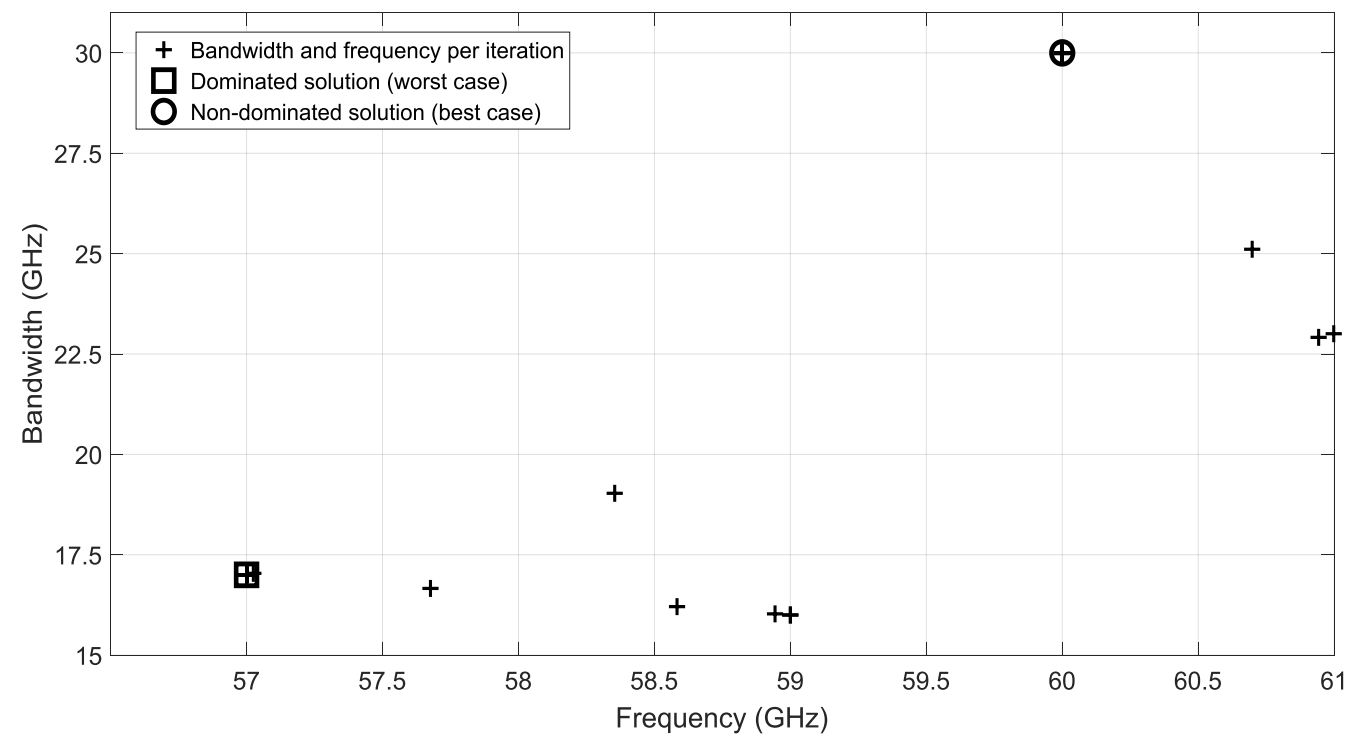

Fig. 1. Relationship of domination for the problem's cost function.

The set of all non-dominated solution define the Pareto's optimal border. Whenever this border is obtained, the "decider" picks the most adequate solution (or tradeoff solution) considering the project's objectives. For simplicity's sake, weighted sums were made to match said objects [4]:

$$
f=\sum_{k=1}^{K} w_{k} f_{k}, \sum_{k=1}^{K} w_{k}=1
$$

The weights are randomly generated according to an uniform distribution, resulting in a sufficient weight variation to guarantee the diversity of solutions and, consequently, the correct approximation in relation to the Pareto Front. The algorithm's adjustable parameters were configured thusly, for MOBA utilization: $\alpha=\gamma=0.9$, NParet $=60$ (number of points in the Pareto Front), $f_{\min }=1.5$ (frequency minimum), $f_{\max }=3.0$ (frequency maximum), $d=2$ (dimension of the search variables), $n=50$ (population size).

Figure 2 demonstrates the fitness evolution for the synthesis process via MOBA. During this process, the cost function's value presents a gradual decrease in relation to its initial value, which denotes greater proximity between the optimal solution for the cost function. The dotted curve illustrates the average (or mean) fitness solution for the entire bat population, and the solid curve represents the best individual solution. 
The algorithm required only 38 iterations to converge to the optimal solution and the total run time of the developed hybrid technique (GRNN+MOBA) was $\cong 327.966 \mathrm{~s}$. Tab. I show some details on the execution time at the main steps of the code. Simulation was performed on a computer with CPU Clock $2.53 \mathrm{GHz}$ and $6 \mathrm{~GB}$ of RAM.

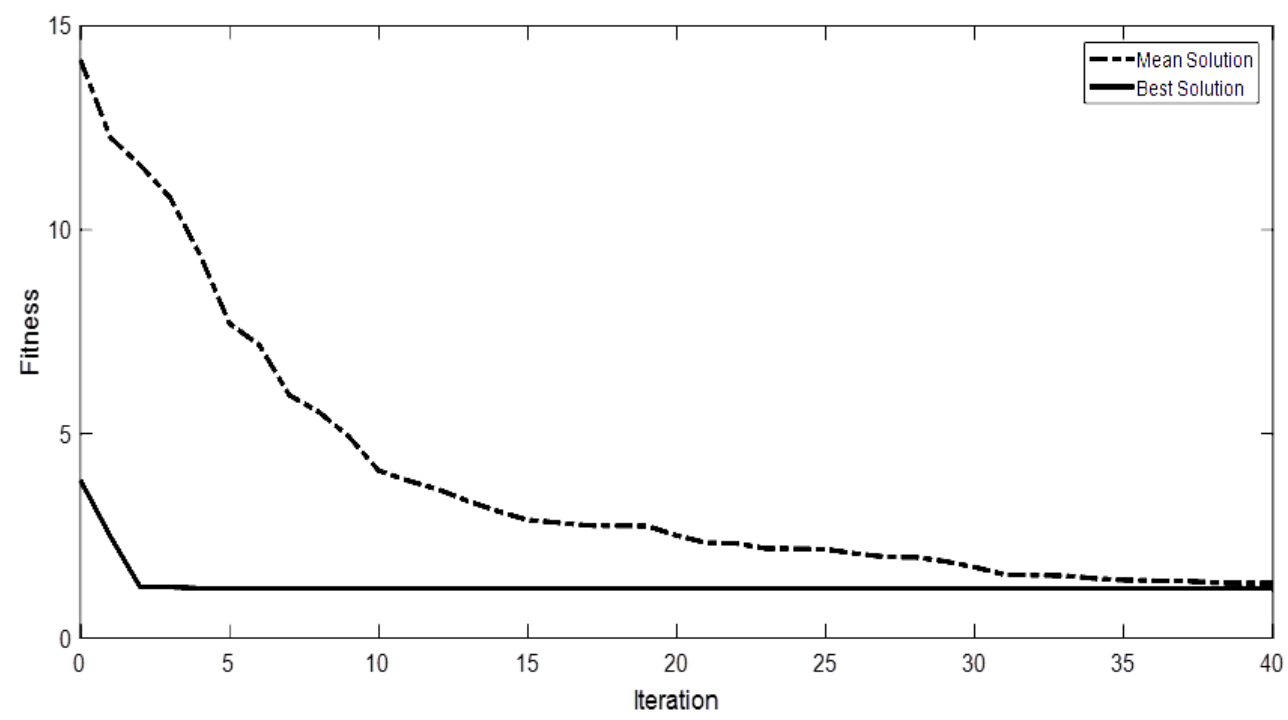

Fig. 2. Fitness evolution in the synthesis process via MOBA, optimized structure for $f_{r}=60.0 \mathrm{GHz}$ and $B W=30.0 \mathrm{GHz}$.

TABLE I. EXECUTION TIME

\begin{tabular}{lcc}
\hline \hline \multicolumn{1}{c}{ Function Name } & CALLS & TOTAL TIME (S) \\
\hline GRNN & 38185 & 30.831 \\
Adjust of the frenquency for each bat & 38185 & 5.338 \\
Search for prey / food & 60 & 291.797 \\
Total Execution Time & - & 327.966 \\
\hline \hline
\end{tabular}

The optimal values returned by MOBA for the diamond-shaped FSS unit cell's dimensions are: Tx $=1.85 \mathrm{~mm}$ and $\mathrm{Ty}=3.7 \mathrm{~mm} ; \mathrm{Wx}=1.75 \mathrm{~mm}$ and $\mathrm{Wy}=1.75 \mathrm{~mm}$.

\section{GRNN IMPLEMENTATION}

In 1964, the Regression Neural Networks were introduced by Nadaraya [27] and Watson [28], and remodeled by Specht in 1991 [14] to execute general regressions (linear or non-linear), originating the General Regression Neural Network (GRNN), derived from Radial Basis Neural Network (RBFN). The GRNN's theoretical foundation is based on the concept of non-parametrical estimate, commonly utilized in statistics [29] - [32].

In this type of network, the need of additional knowledge for a satisfactory adjustment of its input parameters is relatively small, and can be done without any kind of updated data insertion by the programmer [14]. Hence, the GRNN algorithm's sole necessity is the input data for network training, 
discarding the whole process of backpropagation [33]. This is what makes GRNN a very powerful tool to acquire approximation between functions, draw comparisons and predictions of performance on practical systems.

For the project of the diamond-shaped FSS proposed in this study, a GRNN with six first-layer inputs has been utilized, also having a five-neuron hidden layer and two output nodes representing the resonant frequency and desired bandwidth.

The architecture for the developed network is exhibited in Figure 3.

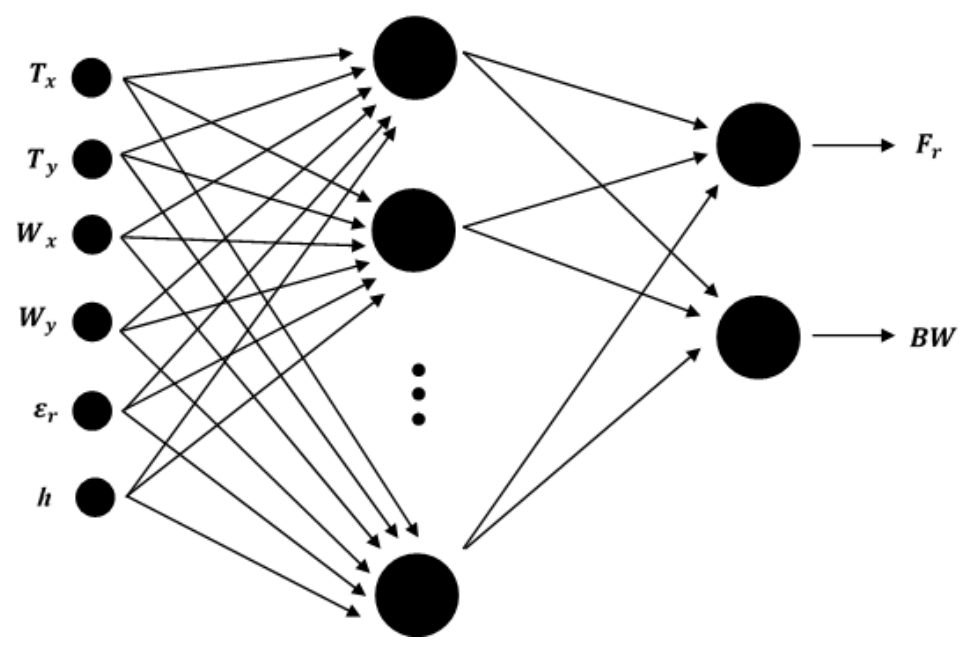

Fig. 3. The utilized GRNN configuration.

For the GRNN model planned for this work, inputs and outputs can be represented as vectors:

$$
\begin{gathered}
x=\left[T_{x}, T_{y}, W_{x}, W_{y}, \varepsilon_{r}, h\right]^{T} \\
y=\left[f_{r}, B W\right]^{T}
\end{gathered}
$$

The GRNN's learning consists of output-based training, which means that the network should respond to the set of inserted restrictions at the entrance, based on the values of $T_{x}$ and $T_{y}-$ unit cell periodicity - and $W_{x}$ and $W_{y}$ - height and width of the patches - that must attend the objectives traced for the project.

On this paradigm, the system is taught to statistically discover prominent characteristics within the input population, thus creating a Region of Interest (RoI) in which the MOBA will conduct searches to find optimal structural data for the diamond-shaped FSS' unit cell.

\section{DIAMOND-SHAPED PATCH FSS}

The last few decades have been marked by a great interest in the use of Frequency Selective Surfaces (FSS), as spatial filters, for several microwave applications [2], [3], [5] - [9]. The FSS are typically two-dimensional periodic arrays, which act as spatial filters [15]. Their frequency behavior depends mainly on the geometry of the elements, the unit cell size, the dielectric material used in the 
manufacture, and the thickness of the substrate [3] - [4]. In addition, they can act as bandpass or band-reject filters, according to the type of the array element, respectively, slot or patch [15].

For this study, a patch-type FSS has been projected. Hence, it is a bandstop filter for applications based on the IEE 802.15.3c standard. In computational simulations, the diamond-shaped patch-type FSS was considered as being built upon the RO4003 substrate, characterized by a relative permittivity $\varepsilon_{r}=3.55$ and dielectric loss tangent $\delta=0.0027$, and the patches were treated as a perfect conductive (PEC) material. Figure 4 (a) and (b) presents the unit cell and device array schematics.

The parameters utilized for the structure's project are shown in Tab. 1, in which is possible to denote how varied the geometrical parameters are for the unit cell, as well as the step-by-step variation programmed into the simulating software.

The structural parameters presented in Tab. II suffered variation to map the operational characteristics on the frequency domain for this structure. The Finite Element Method (FEM) has been applied to the device's electromagnetic (EM) properties. Furtherly, this data has been applied to the training and learning processes of the GRNN.

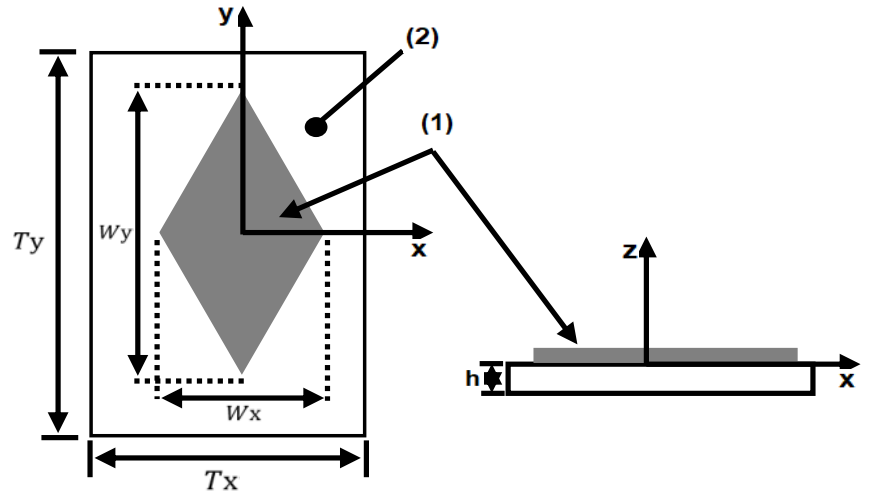

(a)

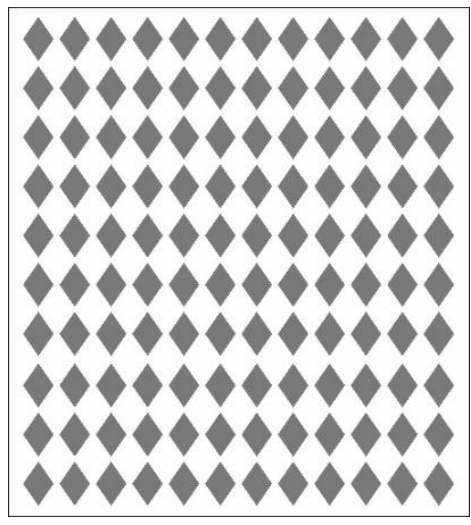

(b)

Fig. 4. Diamond-shaped, patch-type FSS (a) unit cell and (b) the unit cell array, composing the device. The indicator (1) highlights metallization, and (2) highlights the RO4003 substrate.

TABLE II. STRUCTURAL PARAMETERS OF THE DIAMOND-SHAPED FSS

\begin{tabular}{lc}
\hline \hline \multicolumn{1}{c}{ Structural Parameters } & VALUE \\
\hline Array Periodicity $(\mathrm{mm})$ & $T_{x}=[1.85 ; 1.95 ; 2.05 ; 2.15 ; 2.25 ; 2.35]$ \\
Array Periodicity $(\mathrm{mm})$ & $T_{y}=[3.7 ; 3.9 ; 4.1 ; 4.3 ; 4.5 ; 4.7]$ \\
Patch dimensions $(\mathrm{mm})$ & $W_{x}=[1.45 ; 1.55 ; 1.65 ; 1.75]$ \\
Patch dimensions $(\mathrm{mm})$ & $W_{y}=[2.9 ; 3.1 ; 3.3 ; 3.5]$ \\
Substrate height $(\mathrm{mm})$ & $h=0.5$ \\
Substrate relative permittivity & $\varepsilon_{r}=3.55$ \\
\hline \hline
\end{tabular}

\section{HYBRID OPTIMIZATION TECHNIQUE AND RESULTS}

The optimization process built for this paper is divided in two phases: the search phase and the 
analysis phase. The Multiobjective Bat Algorithm (MOBA) is responsible for the search operation and the General Regression Neural Network (GRNN), after training, is responsible for the analysis operation, resulting in a continuous interaction at this phase, as it is illustrated in Fig. 5. Thus, for every new parameter set that the MOBA returns, the GRNN algorithm performs the necessary computation and determines the value of a new dot within the search space inside the Region of Interest (RoI). In this way, the difference between the response given by the network and the values specified for the project is minimized. That is [2] - [3]:

$$
F=\left[f_{1}(x), f_{2}(x)\right]
$$

where,

$$
\begin{gathered}
f_{1}=\left\|f_{r, \text { desired }}-f_{r, \text { obtained }}\right\| \\
f_{2}=\left\|B W_{\text {desired }}-B W_{\text {obtained }}\right\|,
\end{gathered}
$$

In this paradigm the ideal solution would be to find values close or equal to zero for the cost function.

Figure 5 presents entry data referring to the transmission coefficient's scattering matrix of the diamond-shaped patch FSS, according to the modeling parameters exposed in Tab. I and calculated by the Finite Element Method (FEM). In the figure, the cross-like markers highlight EM data that characterize the operation of the structure within the desired frequency band. And in the same figure, it is possible to verify the high-capacity learning and data mapping qualities of the GRNN herein developed, as its circle-like markers surround the crosses.

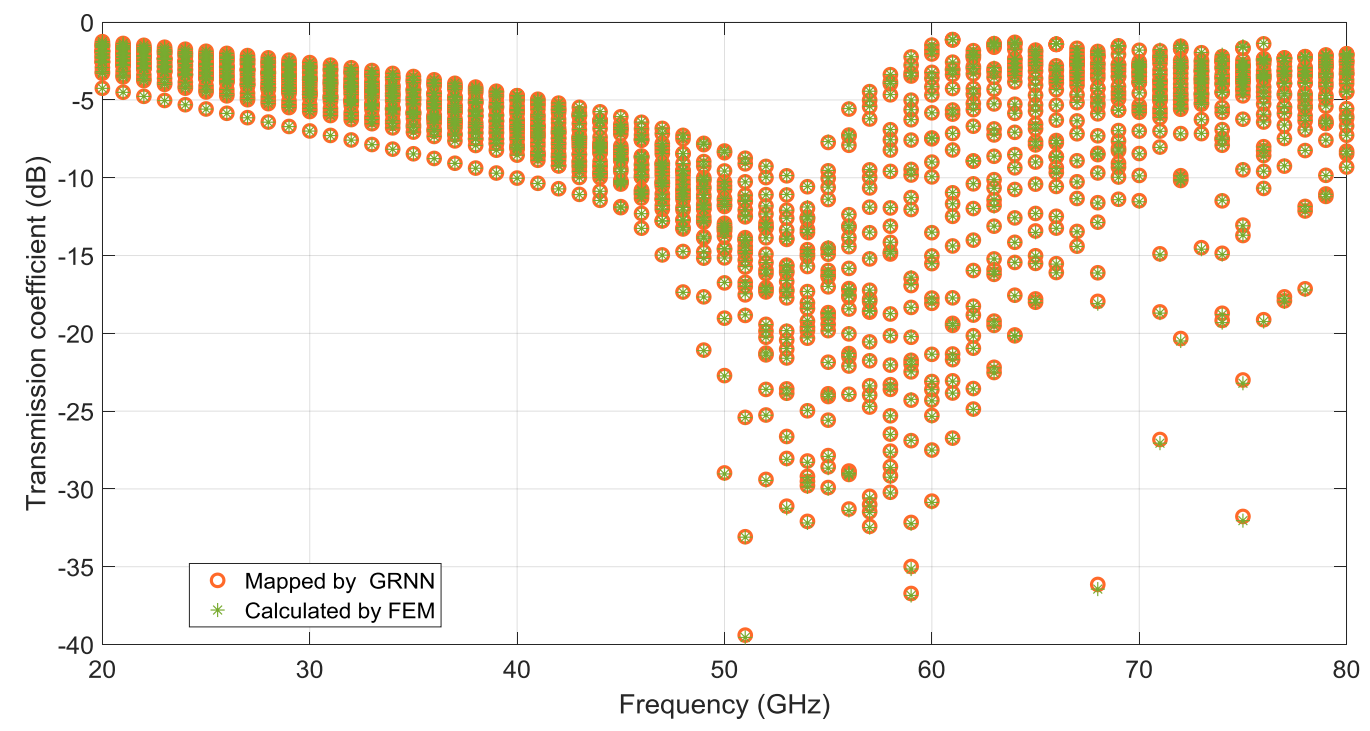

Fig. 5. Learning capacity and input data mapping of the GRNN.

Figure 6 (a) and (b) present results obtained by the GRNN developed for the diamond-shaped, patch-type FSS herein investigated. The dotted lines indicate the network's response, and the other lines, differentiated by symbols, represent the network's training set. 
Journal of Microwaves, Optoelectronics and Electromagnetic Applications, Vol. 18, No. 4, December 2019540 DOI: http://dx.doi.org/10.1590/2179-10742019v18i41729

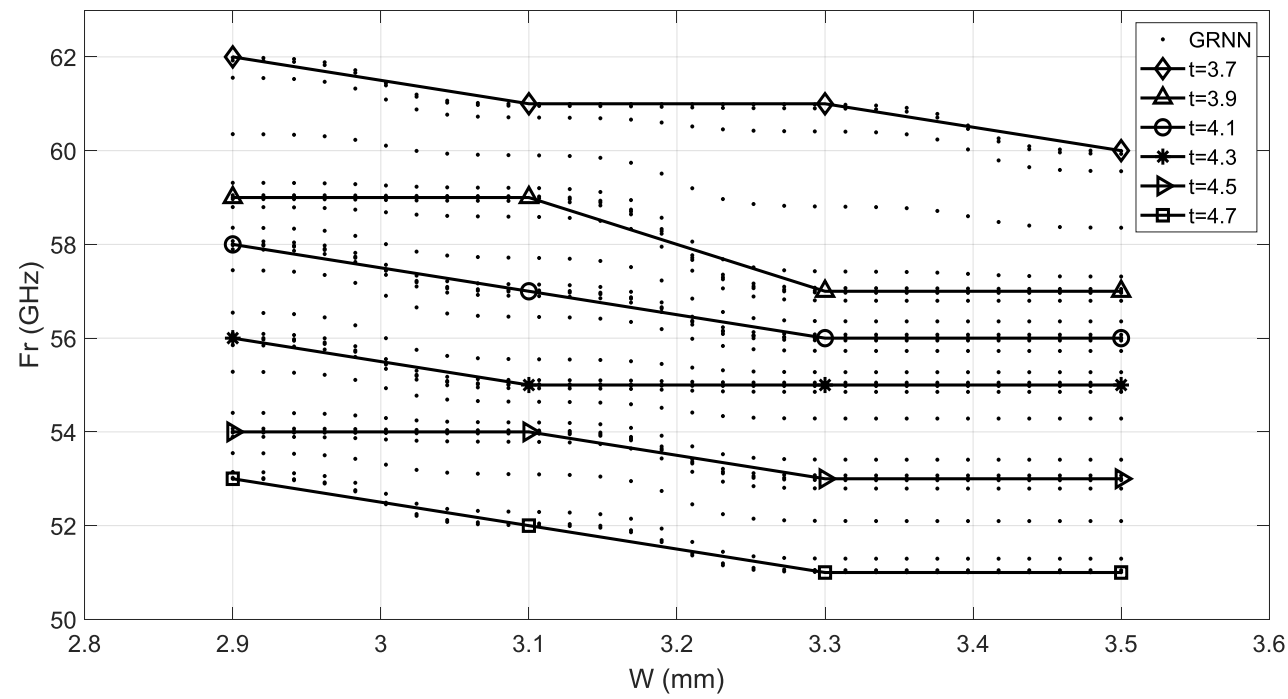

(a)

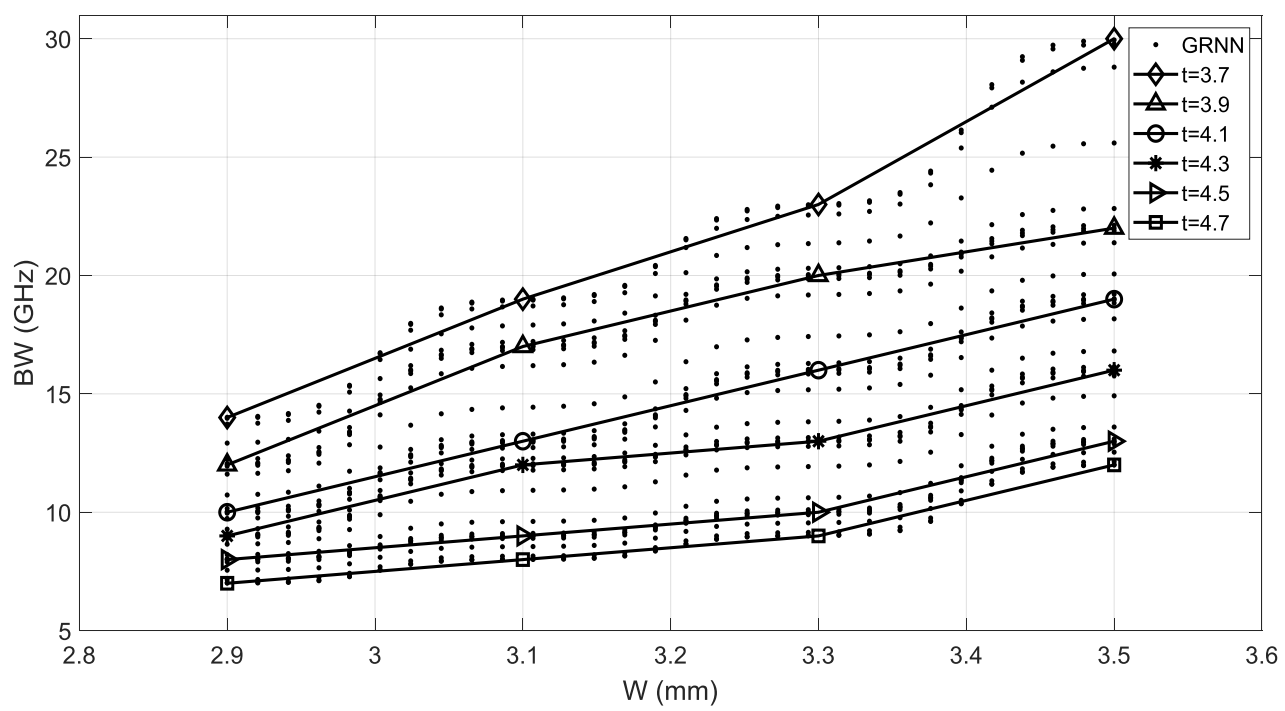

(b)

Fig. 6. GRNN network results for the (a) resonance frequency and (b) bandwidth as a function of the FSS structural parameters $\mathrm{W}$ and $\mathrm{T}$.

By analyzing the response given by the constructed GRNN, it can be denoted that the network demonstrates high learning and input data mapping performance, with entry data provided by FEM (Figure 7) as well as a high capacity of data generalization, as seen in Figure 5 as well as in Figure 
6(a) and (b). This fact assigns greater reliability to the RoI generated so that the MOBA utilize it as a space search for solutions that agree with the established objectives in the cost function.

Figure 7 shows the flowchart of the hybrid technique developed in this study.

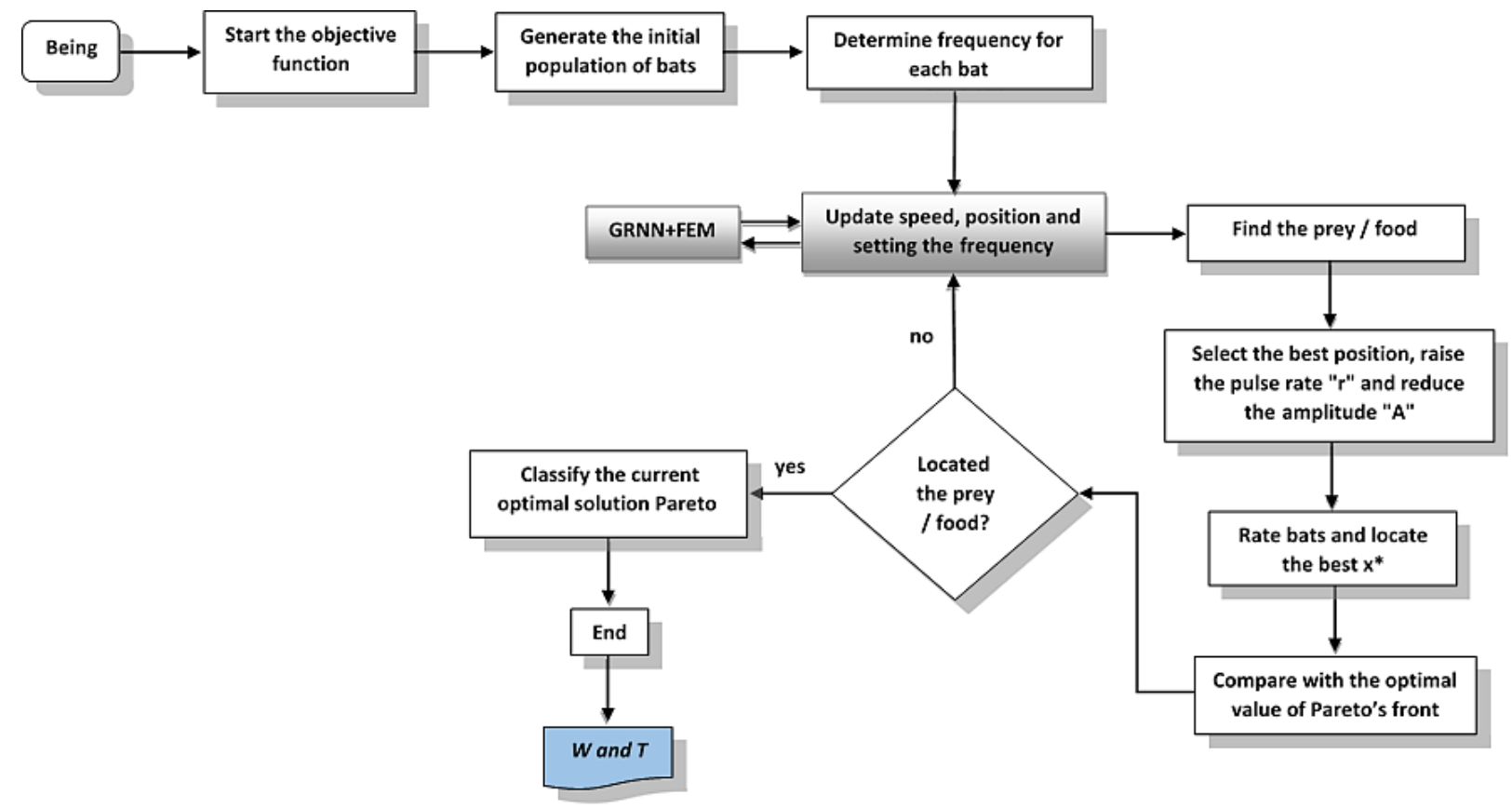

Fig. 7. Flowchart for the hybrid optimization technique applied to the FSS.

Figure 8 shows the simulated with both Finite Element Method (FEM) and Finite Integration Method (FIT), as well as the hybrid technique developed in this study for transmission coefficient in $\mathrm{dB}$ FSS optimized as a function of the resonant frequency $\mathrm{fr}=60.0 \mathrm{GHz}$ and bandwidth $\mathrm{BW}=30.0$ GHz. The optimal structural parameters obtained are $\varepsilon_{r}=3.55 ; \mathrm{h}=0.5 \mathrm{~mm} ; \mathrm{Tx}=1.85 \mathrm{~mm}$ and $\mathrm{Ty}=$ $3.7 \mathrm{~mm} ; \mathrm{Wx}=1.75 \mathrm{~mm}$ and $\mathrm{Wy}=3.5 \mathrm{~mm}$.

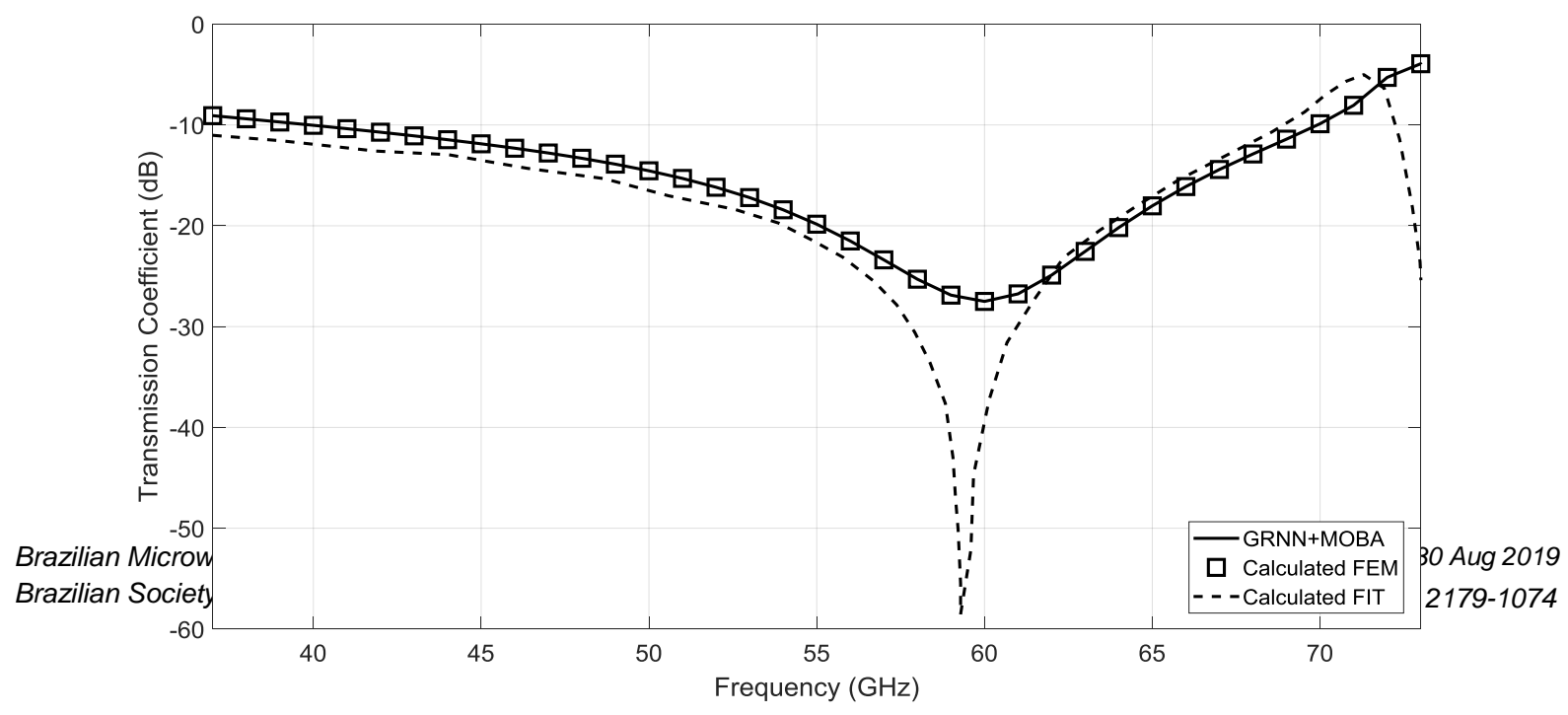


Fig. 8. Transmission coefficient of the diamond-shaped FSS patch with resonant frequency $\mathrm{fr}=60.0 \mathrm{GHz}$ and bandwidth $\mathrm{BW}=30.0 \mathrm{GHz}$, optimized using MOBA. FSS structural parameters are $\varepsilon_{r}=3.55 ; \mathrm{h}=0.5 \mathrm{~mm} ; \mathrm{Tx}=1.85 \mathrm{~mm}$ and $\mathrm{Ty}=$ $3.7 \mathrm{~mm} ; \mathrm{Wx}=1.75 \mathrm{~mm}$ and $\mathrm{Wy}=3.5 \mathrm{~mm}$.

\section{CONCLUSION}

Bioinspired computational techniques aim to better the adaptation of individuals to the environment over the course of many generations, drawing inspiration from natural evolution and selection (e.g. the Generic Algorithm). Analyzing the flight properties of a set of bat species, the Multiobjective Bat Algorithm (MOBA) intends to simulate flight velocity and position of bats, in which they utilize their echolocation sonar device, and applies an optimization that is very similar to the idea of particle swarm intelligence, employed in Particle Swarm Optimization (PSO).

Agreeing with this paradigm, this paper has shown a new bioinspired, multiobjective hybrid optimization technique, associating a General Regression Neural Network (GRNN) with the MOBA, applied to the project and modeling of a Frequency Selective Surface (FSS) with a Diamond-shaped patch elements for mm-Wave filtering applications, specifically, in the IEEE 802.15.3c standard. The developed technique proved to be fast and accurate, see Tab. I, consisting of a more viable tool for the development of radio broadcasting circuits, including planar FSS, filters, and resonators.

In the computational simulations, the periodic array has been considered as being printed upon the isotropic dielectric substrate RO4003. The motives for developing it as such is the simplicity of the investigated geometry, low substrate material cost that is ideal for future prototype fabrication, and allowing operation in an ultra large frequency band. Electromagnetic (EM) data obtained for the training of the GRNN were calculated by complete wave analysis, realized by the Finite Element Method (FEM), aided by the commercial software HFSS. For the validation of results and verification of the developed optimization technique's computational consistency, new simulations has been run using the FEM, and through the numerical Finite Integration Technique (FIT), with the support of the software CST ${ }^{\circledR}$, according to the optimal structural parameters of the FSS' unit cell, and provided by the hybrid optimization algorithm GRNN + MOBA, which are: $W_{y}=3.7 \mathrm{~mm} ; W_{x}=1.85 \mathrm{~mm} ; T_{y}=$ $3.5 \mathrm{~mm}$ and $T_{x}=1.75 \mathrm{~mm}$.

Thereby, a good agreement is observed between all results simulated for both FSS resonance frequency and bandwidth presented in Figure 8. It is important to point out that GRNN-type networks and the MOBA algorithm, when searched about in state-of-the-art literature, had not yet been applied to the FSS optimization process for mm-Wave usage. 
Journal of Microwaves, Optoelectronics and Electromagnetic Applications, Vol. 18, No. 4, December 2019543 DOI: http://dx.doi.org/10.1590/2179-10742019v18i41729

\section{ACKNOWLEDGMENT}

The authors thank the support of CNPq, under covenant 573939/2008-0 (INCT-CSF), CAPES, Group of Telecommunications and Applied Electromagnetism (GTEMA) of the Federal Institute of Education, Science and Technology of Paraíba (IFPB), UFPA and UFRN.

\section{REFERENCES}

[1] X. S. Yang, S. Koziel, "Computational Optimization and Applications in Engineering and Industry," Springer, 2011.

[2] M. C. Alcantara Neto, J. P. L. Araújo, F. J. B. Barros, A. N. Silva, G. P. S. Cavalcante, and A. G. D’Assunção, "Bioinspired multiobjective synthesis of x-band fss via general regression neural network and cuckoo search algorithm," Microwave and Optical Technology Letters, vol. 57, no. 10, pp. 2400-24005, October 2015.

[3] M. C. Alcântara Neto, F. J. B. Barros, J. P. L. Araújo, H. S. Gomes, G. P. S. Cavalcante, A. G. D’Assunção, "A Metaheuristic Hybrid Optimization Technique for Designing Broadband FSS," SBMO/IEEE MTT-S International Microwave and Optoelectronics Conference (IMOC), pp. 1 -5, November 2015.

[4] X. S. Yang, "Bat Algorithm for Multiobjective Optimization," Int. J. Bio-Inspired Computation, vol. 3, no. 5, pp. 267274, September 2011.

[5] R. Saidi, M. Titaouine, A. Djouimaa, T. R. Sousa, A. Gomes Neto, H. Baudrand, "Characterization of Switchable Rectangular Ring FSS with Non Coupled Parallel Metallic Strips for Multi Band and Dual Polarized Applications Using WCIP Method," Journal of Microwaves, Optoelectronics and Electromagnetic Applications, vol. 17, no. 1, pp. 102-120, March 2018.

[6] K. Bencherif, M. Titaouine, R. Saidi, A. Djouimaa, I. Adoui, T. R. Sousa, A. Gomes Neto, H. Baudrand, "Multiband FSS Analysis and Synthesis Based on Parallel Non Coupled Metallic Strips Using WCIP Method," Journal of Microwaves, Optoelectronics and Electromagnetic Applications, vol. 17, no. 4, pp. 433-456, December 2018.

[7] I. Adoui, M. Titaouine1, H. Choutri, R. Saidi, T. R. Sousa, A. Gomes Neto, H. Baudrand, "WCIP Method Applied To Modeling an L-Notched Rectangular Metallic Ring FSS for Multiband Applications and its Equivalent Structure," Journal of Microwaves, Optoelectronics and Electromagnetic Applications, vol. 17, no. 4, pp. 457-476, December 2018.

[8] W. Y. Yong, S. K. A. Rahim, M. Himdi, F. C. Seman, D. L. Suong, M. R. Ramli, and H. A. Elmobarak, "Flexible Convoluted Ring Shaped FSS for X-Band Screening Application," IEEE Access, vol. 6, pp. 11657-11665, March 2018.

[9] Y. Ma, W. Wu , Y. Yuan, W. Yuan, and N. Yuan, "A High-Selective Frequency Selective Surface With Hybrid Unit Cells," IEEE Access, vol. 6, pp. 75259-75267, October 2018.

[10] C. C. Chen, "Transmission through a conducting screen perforated periodically with apertures," IEEE Trans. Microwave Theory Tech., vol. MTT-18, pp. 627-632,1970.

[11] L. Ragan, A. Hassibi, T. S. Rappaport, C. L. Christianson, "Novel on-chip antenna structures and frequency selective surface (FSS) approaches for millimeter wave devices," Vehicular Technology Conference (VTC), 66th IEEE Conference, pp. 2051-2055, 2007.

[12] "Amendment of Parts 215 and 97 of the Commission's Rules to Permit Use of Radio Frequencies Above $40 \mathrm{GHz}$ for New Radio Applications", FCC 95-499 ET Docket no. 94-124 RM-8308, Dec. 1995.

[13] J. P. L. Araújo, J. C. Rodrigues, S. G. C. Fraiha, H. Gomes, G. P. S. Cavalcante, C. e R. L. Francês, "A WLAN planning proposal through computational intelligence and genetic algorithms hybrid approach," The International Conference on Mobile Technology, Applications \& Systems (Mobility Conference), pp. 10-12, Ilan, Taiwan, 2008.

[14] D. F. Specht, “A general regression neural network,” IEEE Transactions on Neural Networks, vol. 2, no. 6, 1991. 
Journal of Microwaves, Optoelectronics and Electromagnetic Applications, Vol. 18, No. 4, December 2019544 DOI: http://dx.doi.org/10.1590/2179-10742019v18i41729

[15] W. C. Araújo, H. W. C. Lins, A. G. D’Assunção Jr., J. L. G. Medeiros and A. G. D’Assunção, “A bioinspired hybrid optimization algorithm for designing broadband frequency selective surfaces," Microwave and Optical Technology Letters, vol. 56, no. 2, 2014.

[16] H. W. C. Lins, E. L. F. Barreto e A. G. D’Assunção, "Enhanced wideband performance of coupled frequency selective surfaces using metaheuristics," Microwave and Optical Technology Letters, vol. 55, no. 4, 2013.

[17] P. H. F. Silva, R. M. S. Cruz e A. G. D’Assunção, "Blending PSO and ANN for optimal design of FSS filters with Koch Island patch elements," IEEE Transactions on Magnetics, vol. 46, no. 8, 2010.

[18] A. Hoorfar, "Evolutionary programming in electromagnetic optimization: a review," IEEE Trans. Antenna and Propag., pp. 523-537, 2007.

[19] G. R. MacCartney, Jr. and T. S. Rappaport, "Study on 3GPP Rural Macrocell Path Loss Models for Millimeter Wave Wireless Communications," in 2017 IEEE International Conference on Communications (ICC), Paris, France, May 2017, pp. 1-7.

[20] S. Sun, G. R. MacCartney Jr., and T. S. Rappaport, "A Novel Millimeter-Wave Channel Simulator and Applications for 5G Wireless Communications," 2017 IEEE International Conference on Communications (ICC), May 2017.

[21] S. Sun, H. Yan, G. R. MacCartney Jr., and T. S. Rappaport, "Millimeter Wave Small-Scale Spatial Statistics in an Urban Microcell Scenario," 2017 IEEE International Conference on Communications (ICC), May 2017.

[22] S. Sun and T. S. Rappaport, "Millimeter Wave MIMO Channel Estimation Based on Adaptive Compressed Sensing," 2017 IEEE International Conference on Communications Workshop (ICCW), May 2017.

[23] J. Ryan, G. R. MacCartney, Jr., and T. S. Rappaport, "Indoor Office Wideband Penetration Loss Measurements at 73 GHz," in 2017 IEEE International Conference on Communications Workshop (ICCW), Paris, France, May 2017, pp. 1-6.

[24] G. R. MacCartney, Jr., H. Yan, S. Sun, and T. S. Rappaport, “A Flexible Wideband Millimeter-Wave Channel Sounder with Local Area and NLOS to LOS Transition Measurements," in 2017 IEEE International Conference on Communications (ICC), Paris, France, May 2017, pp. 1-7.

[25] X. S. Yang, “A new metaheuristic bat-inspired algorithm," Nature Inspired Cooperative Strategies for Optimization, Springer Berlin, vol. 284, pp. 65-74, 2010.

[26] V. Pareto, Cours d'Economie Politique, Rouge, 1886.

[27] E. A. Nadaraya, "On estimating regression,” Theory of Probab. Applicat., vol. 9, pp. 141-142, 1964.

[28] G. S. Watson, "Smooth regression analysis," Sankhya Series A, vol. 26, pp. 359-372, 1964.

[29] L. P. Devroye and L. Györfi, "Nonparametric density estimation: the L_1 view,” U.K.: Wiley, 1983.

[30] R. L. Eubank, "Spline smoothing and nonparametric regression,” New York and Basel: Marcel Dekker, 1988.

[31] L. Rutkowski, "Sequential estimates of probability densities by orthogonal series and their application in pattern classification,” IEEE Trans. Syst., Man, Cybern., vol. SMC-10, no. 12, pp. 918-920, 1980.

[32] L. Rutkowski, "Sequential estimates of a regression function by orthogonal series with applications in discrimination," in Lectures Notes in Statistics, vol. 8. New York, pp. 236-244, 1981.

[33] D. E. Rumelhart, G. E. Hinton, and R.J. Williams, "Learning internal representations by error Propagation,” D. E. Rumelhart, J. L. McClelland and The PDP Research Group, Parallel distributed processing: Explorations in the microstructure of cognition, Foundations, MIT Press, vol. 1, pp. 318-362, Cambridge, MA, 1986. 